\title{
Clinical significance of site-specific metastases in pancreatic cancer: a study based on both clinical trial and real-world data
}

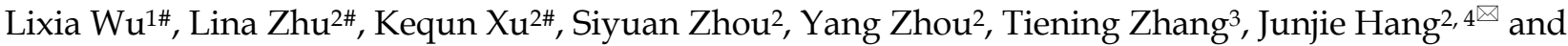 \\ Benny Chung-Ying Zee ${ }^{凶}$ \\ 1. Department of Oncology, Shanghai JingAn District ZhaBei Central Hospital, Shanghai 200070, China. \\ 2. Changzhou No. 2 People's Hospital, Affiliated Hospital of Nanjing Medical University, Changzhou 213000, China. \\ 3. Department of Radiotherapy, Shanghai General Hospital, Shanghai 200080, China. \\ 4. JC School of Public Health and Primary Care, The Chinese University of Hong Kong, Prince of Wales Hospital, Shatin 999077, Hong Kong, China. \\ \#These authors contributed equally to this work. \\ $\bowtie$ Corresponding authors: Junjie Hang, M.D., E-mail: hjj199141@alumni.sjtu.edu.cn; Benny Chung-Ying Zee, Ph.D., E-mail: bzee@cuhk.edu.hk.
}

(c) The author(s). This is an open access article distributed under the terms of the Creative Commons Attribution License (https://creativecommons.org/licenses/by/4.0/). See http://ivyspring.com/terms for full terms and conditions.

Received: 2020.07.05; Accepted: 2020.12.29; Published: 2021.01.18

\begin{abstract}
Background: There is limited consensus on whether metastatic patterns are correlated with prognosis and treatment efficacy in pancreatic cancer. A better understanding of clinical implication of the metastatic patterns is pivotal for therapeutic decision-making and drug development.

Methods: This study included 977 patients with metastatic pancreatic cancer (MPC) in three cohorts. The training cohort included 273 patients from clinical trial NCT00574275 and 367 patients from clinical trial NCT01 124786. As the validation cohort, 337 patients from Changzhou No.2 People's Hospital and Shanghai General Hospital were enrolled. The correlations between different patterns of metastases and clinicopathological characteristics were investigated with the Pearson Chi-Square test. Kaplan-Meier analysis and log-rank test were applied to analyze the survival outcomes among groups with different metastatic patterns. The prognostic value of the number of metastatic sites and other variables was evaluated using the Cox regression model.

Results: MPC patients aged $\geq 65$ years had a higher rate of lung metastasis and those with liver metastasis were prone to have a high level of carbohydrate antigen 19-9 (CA19-9). Additionally, patients with isolated lung metastasis had much better overall survival (OS) than those with isolated liver or peritoneum metastasis. Cox regression analyses showed that the number of metastatic sites was an independent prognostic factor for OS in patients with MPC. Furthermore, for patients with one-site or two-site metastasis, there was a significant difference in OS among patients receiving no chemotherapy, monotherapy and combination therapy. However, for patients with more than two metastatic sites, receiving combination therapy or monotherapy showed limited superiority in OS over receiving no chemotherapy.

Conclusion: MPC patients with isolated lung metastasis had better OS than those with isolated liver or peritoneum metastasis. Moreover, the number of metastatic sites showed prognostic and predictive value in patients with MPC.
\end{abstract}

Key words: lung metastasis; pancreatic cancer; predictive value; prognosis; site-specific metastases

\section{Introduction}

Pancreatic cancer is the seventh leading cause of cancer-related mortality worldwide in both men and women, with an overall five-year survival of less than $6 \%$ [1]. Approximately $80 \%$ of patients with pancreatic cancer are at an advanced stage when they are initially diagnosed [2]. Although marked progress has been made in the treatment of pancreatic cancer, chemotherapy remains the primary treatment for 
patients with metastatic disease. However, only a small proportion of patients benefit from targeted therapy and immunotherapy $[3,4]$.

Liver is the most common site of distant metastases in pancreatic cancer, but the cancer cells can also metastasize to other distant organs [5]. The specific metastatic sites may reflect the molecular background and clinicopathological characteristics of pancreatic cancer subtypes [6-9]. For example, Oweira et al. showed that pancreatic cancer patients with isolated liver metastases had worse survival outcome compared with those with isolated lung or distant nodal metastases [10]. According to the eighth edition of the pancreatic cancer staging published by the American Joint Committee on Cancer (AJCC), pancreatic cancer with distant metastases are defined as M1 [11]. Additionally, only a limited number of studies have investigated the occurrence rate and prognostic value of different metastatic patterns in pancreatic cancer [12-14]. Furthermore, almost no guidelines have taken the number and site of metastases into consideration for the treatment of metastatic pancreatic cancer (MPC).

There has been limited consensus on whether metastatic patterns are correlated with different prognosis and treatment efficacy in pancreatic cancer [15]. However, a better understanding of the metastatic patterns is pivotal for therapeutic decisionmaking and drug development. In this study, we addressed this issue with data from clinical trials and real-world study.

\section{Methods}

\section{Patients}

A total of 273 patients with MPC in clinical trial NCT00574275 and 367 patients with MPC in clinical trial NCT01124786 with complete records of clinicopathological features were selected from the Project Data Sphere (PDS), a not-for-profit initiative allowing collective historical cancer clinical trial data to be shared in public [16]. NCT00574275 was a multinational, double-blind, phase III trial that assessed the efficacy of aflibercept in patients with MPC treated with gemcitabine. All 273 patients were in the comparator arm and were treated with gemcitabine and placebo [17]. NCT01124786 was a multicenter, randomized, phase II trial that compared CO-101 with gemcitabine as first-line therapy in patients with MPC [18]. Among the 367 patients, 182 patients received CO-101 alone and 185 patients received gemcitabine alone. All patients from the two clinical trials were enrolled in the training cohort. Additionally, 337 patients with MPC from Changzhou No. 2 People's Hospital and Shanghai
General Hospital were enrolled in the validation cohort. The following inclusion criteria were applied: (1) pathologically confirmed pancreatic adenocarcinoma; (2) no concurrent cancer at another site; and (3) complete records of clinicopathological features. Ethical approval was obtained by the ethics committees of Changzhou No. 2 People's Hospital.

\section{Statistical analysis}

The Venn plot showing the distribution of patients with different patterns of metastases was generated with the online tool Bioinformatics \& Evolutionary Genomics (http://bioinformatics.psb. ugent.be/webtools/Venn/). Statistical analyses were performed with SPSS statistical software (version 21.0, SPSS Inc., Chicago, IL, USA). Descriptive statistics are presented as median level and 95\% confidence interval $(95 \% \mathrm{CI})$. To assess the correlations between the metastatic sites and clinicopathological characteristics, patients were classified into two groups according to gender (male or female), age ( $<65$ or $\geq 65$ ), Eastern Cooperative Oncology Group performance status (ECOG PS) (0-1 or $\geq 2)$, body mass index (BMI) (normal weight or others), primary tumor location (head and neck or body and tail), and the level of CA19-9 (<1000 or $\geq 1000)$. Comparison between groups was conducted using the Pearson Chi-Square test. Kaplan-Meier analysis and log-rank test were used to evaluate the survival outcome between different groups. The prognostic value of the number of metastatic sites and variables was evaluated by Cox regression model. For each factor, we calculated the hazard ratio (HR) and corresponding 95\%CI. Two-sided $\mathrm{P}<0.05$ was considered statistically significant.

\section{Results}

\section{Patient characteristics}

The baseline clinicopathological characteristics of patients with MPC in both training cohort $(n=640)$ and validation cohort $(n=337)$ are listed in Table 1 . In the training cohort, most patients had a good performance status of 0 to $1(n=623,97.3 \%)$ and $92.5 \%$ were white. Additionally, all patients received gemcitabine or its conjugate CO-101. In the validation cohort, all the patients were Asian and only half had a good performance status of 0 to 1 ( $n=167,49.5 \%)$. In the total validation cohort, $17.2 \%$ of patients received no chemotherapy, $49.9 \%$ received monotherapy and $32.9 \%$ received combination therapy. Liver was the most common metastatic site in both cohorts, with a rate of $83.6 \%(535 / 640)$ in the training cohort and $71.2 \%(240 / 337)$ in the validation cohort. The metastatic rate to lung was similar in the training cohort $(26.3 \%)$ and validation cohort $(26.7 \%)$. A small 
portion of patients also had kidney or adrenal metastases in the training cohort $(n=21,3.3 \%)$ and validation cohort $(n=33,9.8 \%)$. Data on other less common metastatic sites including spleen, bone, soft tissue and brain, as well as the number of metastatic sites in the training cohort and validation cohort, are presented in Supplementary Figure 1. The distribution of patients with different patterns of metastases in the training cohort is shown in Venn plots (Figure 1).

Table 1. Baseline clinicopathological characteristics of patients with MPC

\begin{tabular}{|c|c|c|c|}
\hline & $\begin{array}{l}\text { Training cohort } 1 \\
(\mathrm{n}=273)\end{array}$ & $\begin{array}{l}\text { Training cohort } 2 \\
(\mathrm{n}=367)\end{array}$ & $\begin{array}{l}\text { Validation } \\
\text { cohort }(n=337)\end{array}$ \\
\hline Age (years) & $61(34-84)$ & $62(26-86)$ & $63(27-89)$ \\
\hline \multicolumn{4}{|l|}{ Gender } \\
\hline Male & $156(57.1 \%)$ & $219(59.7 \%)$ & $208(61.7 \%)$ \\
\hline Female & $117(42.9 \%)$ & $148(40.3 \%)$ & $129(38.3 \%)$ \\
\hline \multicolumn{4}{|l|}{ Performance status } \\
\hline ECOG PS $=0$ & $98(35.9 \%)$ & $76(20.7 \%)$ & $23(6.8 \%)$ \\
\hline ECOG PS=1 & $159(58.2 \%)$ & $290(79.0 \%)$ & $144(42.7 \%)$ \\
\hline ECOG PS=2 & $16(5.9 \%)$ & $1(0.3 \%)$ & $151(44.8 \%)$ \\
\hline ECOG PS=3 & 0 & 0 & $19(5.6 \%)$ \\
\hline \multicolumn{4}{|l|}{ Body mass index } \\
\hline Underweight $(<18.5)$ & $16(5.9 \%)$ & $24(6.5 \%)$ & $65(19.3 \%)$ \\
\hline $\begin{array}{l}\text { Normal weight } \\
(18.5-25)\end{array}$ & $146(53.5 \%)$ & $199(54.2 \%)$ & $240(71.2 \%)$ \\
\hline Overweight (25-30) & $80(29.3 \%)$ & $98(26.7 \%)$ & $31(9.2 \%)$ \\
\hline Obese $(\geq 30)$ & $31(11.4 \%)$ & $36(9.8 \%)$ & $1(0.3 \%)$ \\
\hline Missing & 0 & $10(2.7 \%)$ & 0 \\
\hline \multicolumn{4}{|l|}{ Race } \\
\hline Asian & 0 & $5(1.4 \%)$ & $337(100 \%)$ \\
\hline White & $265(97.1 \%)$ & $327(89.1 \%)$ & 0 \\
\hline Black & 0 & $3(0.8 \%)$ & 0 \\
\hline Others & $8(2.9 \%)$ & $13(3.5 \%)$ & 0 \\
\hline Missing & 0 & $19(5.2 \%)$ & 0 \\
\hline \multicolumn{4}{|c|}{ Primary tumor location } \\
\hline Head and neck & $115(42.1 \%)$ & 0 & $146(43.3 \%)$ \\
\hline Body and tail & $86(31.5 \%)$ & 0 & $190(56.4 \%)$ \\
\hline Entire pancreas & $72(26.4 \%)$ & 0 & $1(0.3 \%)$ \\
\hline Unknown & 0 & $367(100 \%)$ & 0 \\
\hline \multicolumn{4}{|l|}{ Metastatic site } \\
\hline Liver & $214(78.4 \%)$ & $321(87.5 \%)$ & $240(71.2 \%)$ \\
\hline Lung & $67(24.5 \%)$ & $101(27.5 \%)$ & $90(26.7 \%)$ \\
\hline Peritoneum & $64(23.4 \%)$ & $74(20.2 \%)$ & $100(29.7 \%)$ \\
\hline Kidney or adrenal & $15(5.5 \%)$ & $6(1.6 \%)$ & $33(9.8 \%)$ \\
\hline Lymph nodes & $124(45.4 \%)$ & $193(52.6 \%)$ & $172(51.0 \%)$ \\
\hline Others & $24(8.7 \%)$ & $92(25.1 \%)$ & $70(20.8 \%)$ \\
\hline \multicolumn{4}{|l|}{ Chemotherapy } \\
\hline No & 0 & 0 & $58(17.2 \%)$ \\
\hline Monotherapy & $273(100 \%)$ & $367(100 \%)$ & $168(49.9 \%)$ \\
\hline Combination therapy & 0 & 0 & $111(32.9 \%)$ \\
\hline CA $19-9(\mathrm{U} / \mathrm{mL})$ & $1081.0(0.6-1743408.0)$ & $2525.0(0-2060000.0)$ & $453.5(0-3500.0)$ \\
\hline Missing & $14(5.1 \%)$ & $33(9.0 \%)$ & $5(1.5 \%)$ \\
\hline
\end{tabular}

\section{Correlations between clinicopathological characteristics and metastatic sites}

In the training cohort, patients aged $<65$ years were more prone to have liver metastases $(\mathrm{P}=0.009)$ while patients aged $\geq 65$ years had a higher metastatic rate of lung ( $\mathrm{P}=0.027$ ) (Table 2). Additionally, patients with abnormal BMI more frequently showed lung metastasis $(\mathrm{P}=0.040)$. Moreover, patients with the primary tumor location in body and tail had higher rate of peritoneum metastasis $(\mathrm{P}=0.023)$. Intriguingly, CA19-9 $\geq 1000 \mathrm{U} / \mathrm{ml}$ was significantly correlated with liver metastases $(\mathrm{P}=0.001)$. Supplementary Table 1 showed that patients aged $\geq 65$ also had a higher metastatic rate in lung $(\mathrm{P}=0.043)$ and patients with liver metastasis were more prone to have high levels of CA19-9 $(\mathrm{P}=0.013)$ in the validation cohort. Nevertheless, no difference in other characteristics was observed between patients with or without metastases in liver, lung or peritoneum.

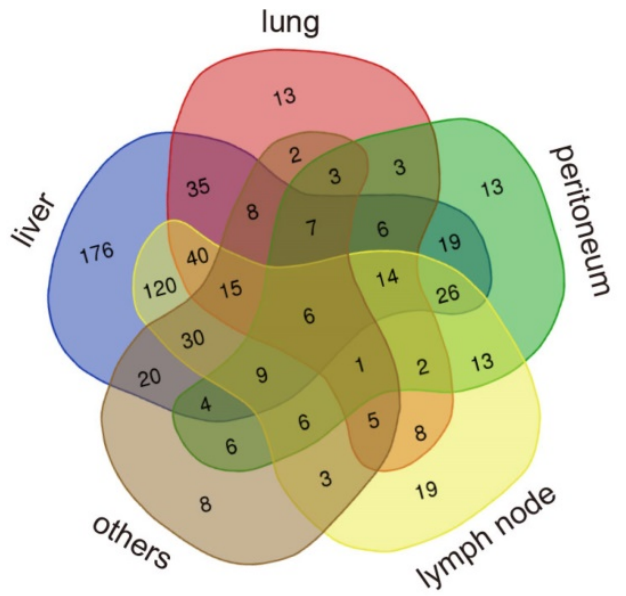

Figure 1. The distribution of patients with different patterns of metastases.

Table 2. Clinicopathological characteristics according to metastatic sites in the training cohort

\begin{tabular}{|c|c|c|c|c|c|c|c|c|c|}
\hline \multirow[t]{2}{*}{ Characteristics } & \multicolumn{2}{|c|}{$\begin{array}{l}\text { Liver } \\
\text { metastasis }\end{array}$} & \multirow[t]{2}{*}{$P$} & \multicolumn{2}{|c|}{$\begin{array}{l}\text { Lung } \\
\text { metastasis }\end{array}$} & \multirow[t]{2}{*}{$P$} & \multicolumn{2}{|c|}{$\begin{array}{l}\text { Peritoneum } \\
\text { metastasis }\end{array}$} & \multirow[t]{2}{*}{$P$} \\
\hline & Yes & No & & Yes & No & & Yes & No & \\
\hline \multicolumn{10}{|l|}{ Gender } \\
\hline Male & 317 & 58 & 0.445 & 92 & 283 & 0.240 & 77 & 298 & 0.451 \\
\hline Female & 218 & 47 & & 76 & 189 & & 61 & 204 & \\
\hline \multicolumn{10}{|l|}{ Age } \\
\hline$<65$ & 343 & 53 & 0.009 & 92 & 304 & 0.027 & 83 & 313 & 0.637 \\
\hline$\geq 65$ & 192 & 52 & & 76 & 168 & & 55 & 189 & \\
\hline \multicolumn{10}{|l|}{ ECOG PS } \\
\hline $0-1$ & 522 & 101 & 0.502 & 134 & 489 & 0.770 & 160 & 463 & 0.088 \\
\hline$\geq 2$ & 13 & 4 & & 4 & 13 & & 8 & 9 & \\
\hline \multicolumn{10}{|c|}{ Body mass index } \\
\hline $\begin{array}{l}\text { Normal } \\
\text { weight }\end{array}$ & 290 & 55 & 0.761 & 78 & 267 & 0.040 & 79 & 266 & 0.379 \\
\hline Others & 237 & 48 & & 85 & 200 & & 57 & 228 & \\
\hline \multicolumn{10}{|c|}{ Tumor location } \\
\hline Head and neck & 87 & 28 & 0.108 & 25 & 90 & 0.952 & 16 & 99 & 0.023 \\
\hline Body and tail & 73 & 13 & & 67 & 17 & & 23 & 63 & \\
\hline \multicolumn{10}{|c|}{ CA19-9 (U/ml) } \\
\hline$<1000$ & 202 & 54 & 0.001 & 67 & 189 & 0.922 & 54 & 202 & 0.800 \\
\hline$\geq 1000$ & 299 & 38 & & 87 & 250 & & 74 & 263 & \\
\hline
\end{tabular}

\section{Survival outcome among liver, lung and peritoneum metastasis groups}

In the training cohort, the median OS of patients with isolated lung metastasis was significantly longer than that of patients with isolated liver metastasis $(\mathrm{P}=0.044)$ or peritoneum metastasis $(\mathrm{P}=0.041$, Figure 2A). However, no significant difference was identified in OS between patients with isolated liver metastasis 
and those with peritoneum metastasis $(\mathrm{P}=0.369)$. For two-site metastases, there were also no significant differences in OS among patients with liver and lung, liver and peritoneum, and lung and peritoneum metastases (all $\mathrm{P}>0.05$, Figure $2 \mathrm{~B}$ ). Similar results were observed in the validation cohort (Figure 2C and 2D).

\section{Prognostic value of the number of metastatic sites}

As shown in the outer ring of Figure 3A, there were $229(35.8 \%)$ patients with isolated metastases, 229 (35.8\%) patients with two-site metastases, 126 $(19.7 \%)$ patients with three-site metastases, $50(7.8 \%)$ patients with four-site metastases and $6(0.9 \%)$ patients with five-site metastases in the training cohort. As shown in the inner ring of Figure 3A, there were $103(30.6 \%)$ patients with isolated metastases, $129(38.3 \%)$ patients with two-site metastases, 72 (21.4\%) patients with three-site metastases, 25 (7.4\%) patients with four-site metastases and $8(2.4 \%)$ patients with five-site metastases in the validation cohort.

In the training cohort, patients with two-site

A

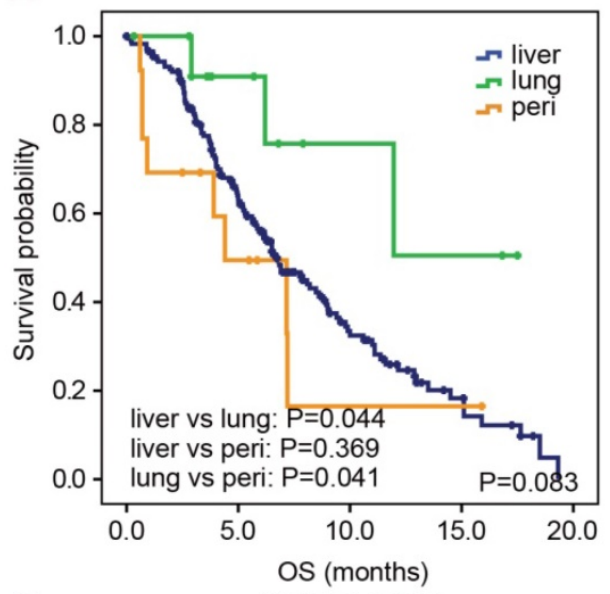

C

Training cohort

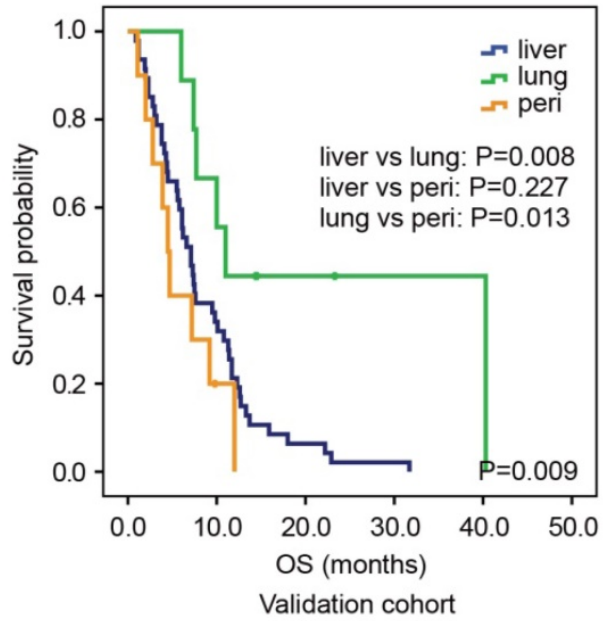

metastases showed the tendency of having poorer OS than patients with one metastatic site (6.4 months vs. 7.6 months, $\mathrm{P}=0.058$ ) but exhibited better OS than those with three metastatic sites (6.4 months vs. 5.1 months, $\mathrm{P}=0.010$ ) and those with more than three metastatic sites (6.4 months vs. 4.5 months, $\mathrm{P}=0.013$ ). However, no significant difference was observed between the OS of patients with three metastatic sites and those with more than three metastatic sites (5.1 months vs. 4.5 months, $\mathrm{P}=0.587$, Figure $3 \mathrm{~B}$ ). Similar results were observed in the validation cohort (Figure 3C). Patients with two-site metastases had poorer OS than those with one metastatic site (5.6 months vs 7.9 months, $\mathrm{P}=0.001$ ) but had better OS than those with three-site metastases (5.6 months vs 4.1 months, $\mathrm{P}=0.023$ ) and those with more than three metastatic sites (5.6 months vs 4.4 months, $\mathrm{P}=0.006$ ). Likewise, there was no significant difference in OS between patients with three metastatic sites and those with more than three metastatic sites (4.1 months vs 4.4 months, $\mathrm{P}=0.392$ ).

B
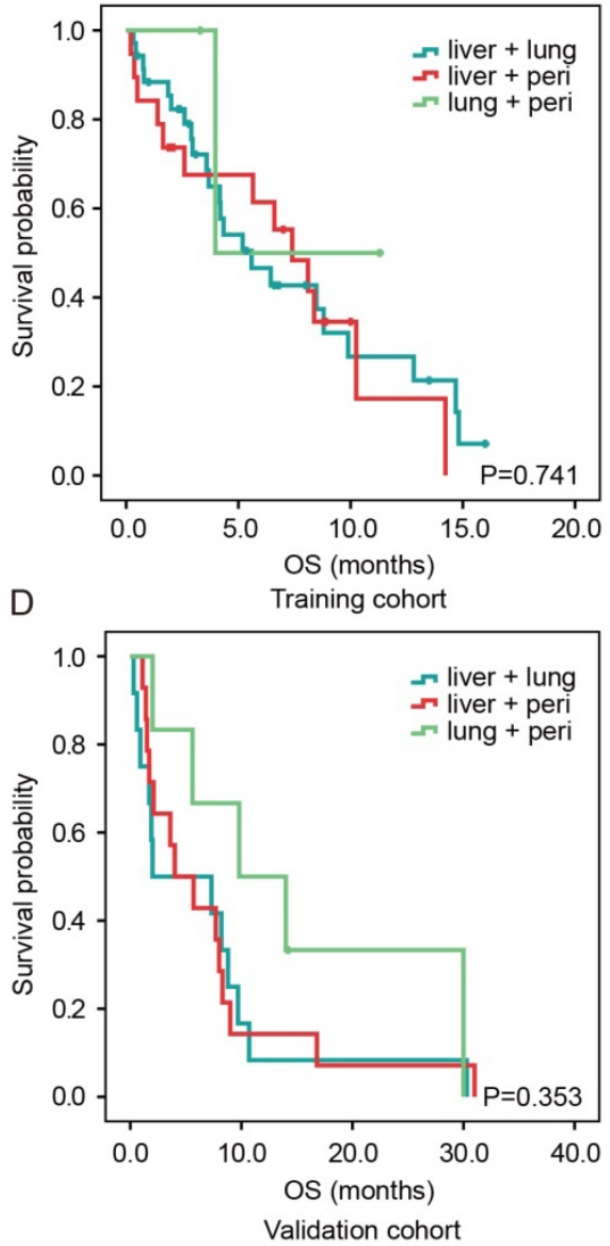

Figure 2. Kaplan-Meier estimates OS according to different metastatic patterns in both the training cohort (A, B) and validation cohort (C,D). Abbreviation: peri, peritoneum. 

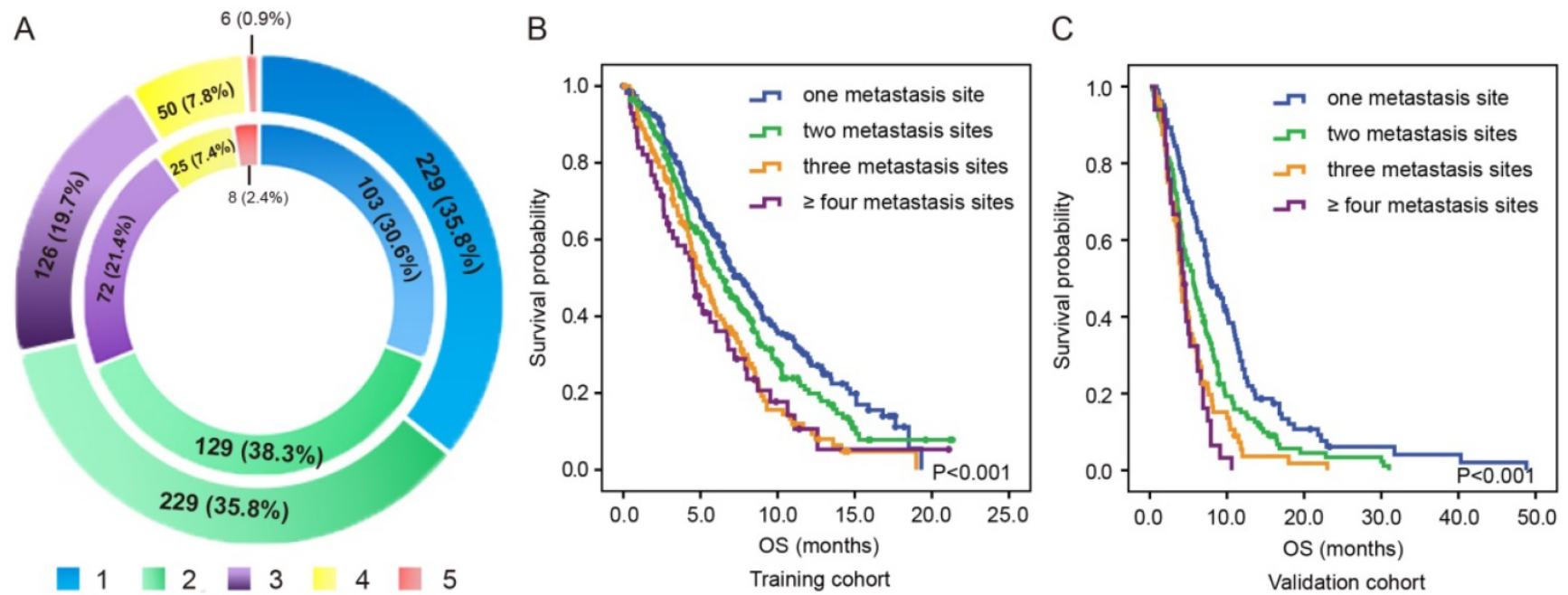

Number of metastatic sites

Figure 3. The distribution $(A)$ and survival outcome according to the number of metastatic sites in both the training cohort (B) and validation cohort (C).

A

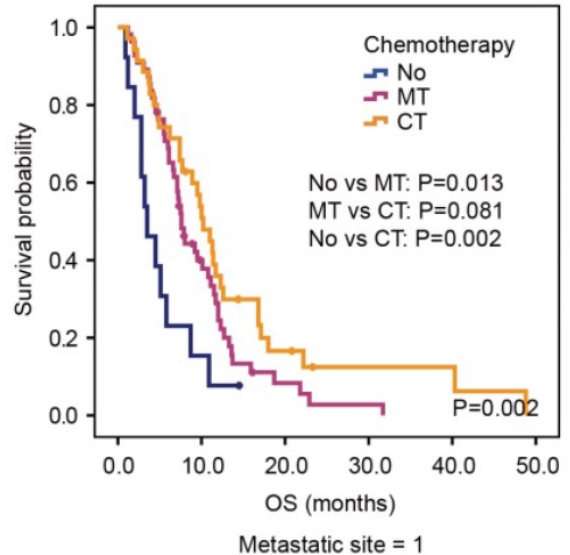

B

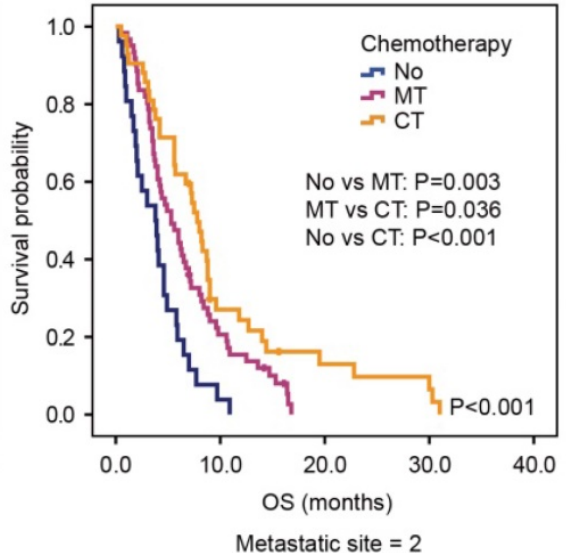

C

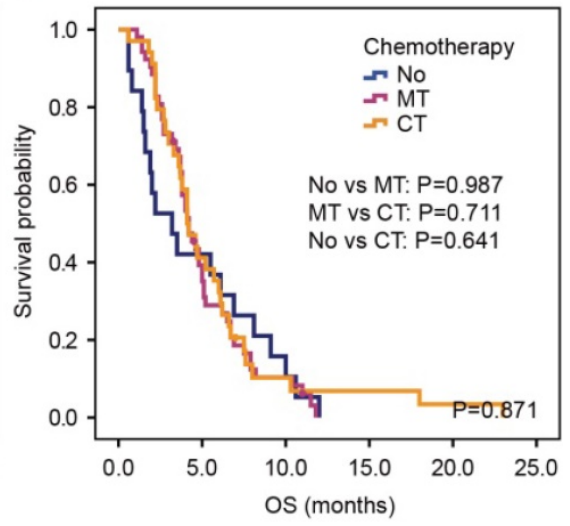

Metastatic site $\geq 3$

Figure 4. Kaplan-Meier estimates OS according to chemotherapy in the group of patients with one metastatic site (A), two metastatic sites (B) and more than two metastatic sites (C) in the validation cohort. Abbreviation: No, no chemotherapy; MT, monotherapy; CT, combination therapy.

In univariate analysis, ECOG PS, CA19-9 and the number of metastatic sites were all significantly correlated with OS. All of these factors were subsequently analyzed in multivariate analysis, and all three factors showed independent prognostic value in the training cohort (Table 3). Because of the heterogeneity in outcomes of chemotherapy for patients in the validation cohort, we included chemotherapy into analysis by classifying patients into three groups: no chemotherapy, monotherapy and combination therapy. In univariate analysis, ECOG PS, CA19-9, number of metastatic sites and chemotherapy were significantly correlated with OS. However, only the number of metastatic sites and chemotherapy showed independent prognostic value in multivariate analysis (Supplementary Table 2).

\section{The predictive value of metastatic sites in palliative treatment}

We next investigated whether the number of metastatic sites affected the efficacy of chemotherapy by dividing patients into three groups: patients with one-site metastasis, patients with two-site metastasis and patients with more than two metastatic sites. In the first group, patients receiving monotherapy or combination therapy showed better OS than those receiving no chemotherapy $(\mathrm{P}=0.002$, Figure $4 \mathrm{~A})$. Furthermore, a trend was seen that combination therapy was superior compared with monotherapy in this group of patients $(\mathrm{P}=0.081)$. Likewise, for patients with two-site metastasis, there was a significant difference among those receiving no chemotherapy, monotherapy and combination therapy (3.8 months vs. 5.3 months vs. 7.8 months, $\mathrm{P}<0.001$, Figure $4 \mathrm{~B}$ ). However, for patients with more than two metastatic sites, patients receiving combination therapy or monotherapy showed no superiority in OS than patients receiving no chemotherapy (4.1 months vs. 4.2 months vs. 3.2 months, $P=0.871$, Figure 4 C). 
Table 3. Univariate and multivariate analysis of prognostic factors for OS in the training cohort

\begin{tabular}{|c|c|c|c|c|c|c|}
\hline \multirow[t]{2}{*}{ Characteristics } & \multicolumn{3}{|c|}{ Univariate analysis } & \multicolumn{3}{|c|}{ Multivariate analysis } \\
\hline & HR & $95 \% \mathrm{CI}$ & $P$ & HR & $95 \% \mathrm{CI}$ & $P$ \\
\hline \multicolumn{7}{|l|}{ Gender } \\
\hline Male & Ref & & & & & \\
\hline Female & 0.998 & 0.907-1.097 & 0.965 & & & \\
\hline \multicolumn{7}{|l|}{ Age } \\
\hline$<65$ & Ref & & & & & \\
\hline$\geq 65$ & 1.072 & $0.886-1.298$ & 0.474 & & & \\
\hline \multicolumn{7}{|l|}{ ECOG PS } \\
\hline 0 & Ref & & & Ref & & \\
\hline 1 & 1.702 & $1.361-2.128$ & $<0.001$ & 1.617 & $1.279-2.044$ & $<0.001$ \\
\hline 2 & 2.075 & 1.139-3.781 & 0.017 & 1.650 & 0.874-3.115 & 0.122 \\
\hline \multicolumn{7}{|c|}{ Body mass index } \\
\hline Normal weight & Ref & & & & & \\
\hline Others & 1.007 & $0.833-1.218$ & 0.939 & & & \\
\hline \multicolumn{7}{|c|}{ Primary tumor location } \\
\hline Head and neck & Ref & & & & & \\
\hline Body and tail & 1.195 & $0.819-1.744$ & 0.356 & & & \\
\hline \multicolumn{7}{|l|}{ CA19-9 (U/ml) } \\
\hline$<1000$ & Ref & & & Ref & & \\
\hline$\geq 1000$ & 1.638 & $1.337-2.005$ & $<0.001$ & 1.557 & $1.268-1.913$ & $<0.001$ \\
\hline \multicolumn{7}{|c|}{ Number of metastatic sites } \\
\hline One & Ref & & & Ref & & \\
\hline Two & 1.246 & $0.991-1.568$ & 0.060 & 1.230 & $0.966-1.565$ & 0.093 \\
\hline Three & 1.734 & $1.339-2.246$ & $<0.001$ & 1.734 & $1.329-2.263$ & $<0.001$ \\
\hline More than three & 1.887 & $1.342-2.653$ & $<0.001$ & 1.814 & $1.266-2.600$ & 0.001 \\
\hline
\end{tabular}

\section{Discussion}

The survival rate of MPC is very dismal, but few studies have examined the survival rate and treatment effect in view of the number and sites of metastases. The main findings of this study were the following: (1) patients aged $\geq 65$ years had a higher metastatic rate of lung than those aged $<65$ years and patients with liver metastasis were prone to have high level of CA19-9; (2) patients with isolated lung metastasis had much better OS than those with isolated liver or peritoneum metastasis; (3) the number of metastatic sites was an independent prognostic factor for OS in patients with MPC and (4) for patients with one-site or two-site metastasis, there was a significant difference in OS among those receiving no chemotherapy, monotherapy and combination therapy, while for patients with more than two metastatic sites, combination therapy or monotherapy showed no superiority in OS over best support care.

Pancreatic cancer can metastasize to different organs by lymphogenic, hematogenous and perineural route or by direct spread [19]. In a large-scale autopsy study in pancreatic cancer, liver was the most common metastatic site, followed by distant lymph node, lung and peritoneum. Other less common metastatic sites include kidney, adrenal, bone, spleen, gallbladder, omentum and, brain [5]. Our work demonstrated a similar distribution of metastatic pattern, as shown in Table 1.
Intriguingly, we found that patients with liver metastasis were prone to have a high level of CA19-9. Because CA19-9 is usually considered as a measure of pancreatic tumor burden, it is reasonable that patients with higher tumor burden will have an increased chance to develop liver metastasis. The level of CA19-9 is also determined by Lewis antigens and associated genes like a1-2 fucosyltransferase (FUT2) and a1-4 fucosyltransferase (FUT3) genes [20,21]. However, there is still no evidence to suggest that the expression of these genes is correlated with liver metastasis.

In a case series, Deeb et al. observed a longer than expected survival in five pancreatic cancer patients with pulmonary metastases [22]. Katz et al. found that the most common metastatic site was the lung among long-term survivors with pancreatic cancer [23]. More recently, Lovecek et al. found that pancreatic adenocarcinoma patients with metachronous pulmonary metastases had a better OS of 31.81 months [6]. In our work, patients with isolated lung metastasis also had much better OS than those with isolated liver or peritoneal metastasis in both the training cohort and validation cohort. Emerging evidence has suggested that surgery should be considered for patients with isolated lung oligometastases $[6,7,19,24]$. However, our work demonstrated that patients with age $\geq 65$ had a much higher metastatic rate of lung than those with age $<65$. As old age usually means higher risk and more difficulty in recovering from interventions like surgery, individual evaluation should be carefully conducted for these patients.

Previous studies showed that the numbers of distant metastases were significantly correlated with outcomes in some types of cancer $[25,26]$, but a similar correlation was not seen in pancreatic cancer. Oweira et al. found no significant difference in OS between pancreatic cancer patients with single metastasis and multiple metastases $(\mathrm{P}=0.409)$ [10]. Intriguingly, in our work, the number of metastatic sites was an independent prognostic factor for OS in patients with MPC. These findings may suggest that the number of metastatic sites plays a more important role than the number of distant metastases in patient' outcome. Moreover, with the data from the real-world study, we found that for patients with one or two-site metastasis, the OS of patients receiving combination therapy was much longer than those receiving monotherapy or no chemotherapy. However, for patients with more than two metastatic sites, receiving combination therapy or monotherapy showed no superiority in OS than receiving no chemotherapy. Because of the small sample size and the retrospective design of this study, more studies 
are warranted to investigate whether other interventions, like chemotherapy, are necessary for MPC patients with more than two metastatic sites.

This study has several limitations. First, there was an evident heterogeneity in the data source and race of patients in the training cohort and validation cohort. Second, the relatively small sample size for every metastatic pattern was likely to make the results less robust. Third, other factors, such as primary tumor size, living habit and comorbidities, were not included in the analysis, which may lead to unexpected bias. Additionally, we did not take the number of metastases in each metastatic site into consideration because of the limitation of the data source. Thus, further research is needed to verify and expand on the clinical significance of site-specific metastases in pancreatic cancer.

In conclusion, MPC patients with isolated lung metastasis had better OS than those with isolated liver or peritoneum metastasis. Additionally, the number of metastatic sites showed both prognostic and predictive value in patients with MPC.

\section{Abbreviations}

MPC: metastatic pancreatic cancer; CA19-9: carbohydrate antigen 19-9; OS: overall survival; AJCC: American Joint Committee on Cancer; PDS: Project Data Sphere; 95\%CI: 95\% confidence interval; ECOG PS: Eastern Cooperative Oncology Group performance status; BMI: body mass index; HR: hazard ratio; FUT2: a1-2 fucosyltransferase; FUT3: a1-4 fucosyltransferase.

\section{Supplementary Material}

Supplementary figures and tables. http://www.jcancer.org/v12p1715s1.pdf

\section{Acknowledgements}

This work was supported by grants from the National Natural Science Foundation of China (81902955), Natural Science Foundation of Jiangsu Province (BK20190161), Project of Jiangsu Shuangchuang Doctor (QT201904), Foundation of Changzhou Sci \& Tech Program (CJ20190096), the Youth Science and Technology Project of Changzhou Health and Family Planning Commission (QN201817).

\section{Competing Interests}

The authors have declared that no competing interest exists.

\section{References}

1. Bray F, Ferlay J, Soerjomataram I, et al. Global cancer statistics 2018: GLOBOCAN estimates of incidence and mortality worldwide for 36 cancers in 185 countries. CA Cancer J Clin. 2018; 68: 394-424.
2. Kamisawa T, Wood LD, Itoi T, et al. Pancreatic cancer. Lancet. 2016; 388: 73-85.

3. Golan T, Hammel P, Reni M, et al. Maintenance Olaparib for Germline BRCAMutated Metastatic Pancreatic Cancer. N Engl J Med. 2019; 381: 317-327.

4. Lambert A, Schwarz L, Borbath I, et al. An update on treatment options for pancreatic adenocarcinoma. Ther Adv Med Oncol. 2019; 11: 1758835919875568 .

5. Disibio G, French SW. Metastatic patterns of cancers: results from a large autopsy study. Arch Pathol Lab Med. 2008; 132: 931-939.

6. Lovecek M, Skalicky P, Chudacek J, et al. Different clinical presentations of metachronous pulmonary metastases after resection of pancreatic ductal adenocarcinoma: Retrospective study and review of the literature. World J Gastroenterol. 2017; 23: 6420-6428.

7. Matsuki R, Sugiyama M, Takei $H$, et al. Long-term survival with repeat resection for lung oligometastasis from pancreatic ductal adenocarcinoma: a case report. Surg Case Rep. 2018; 4: 26.

8. Reichert M, Bakir B, Moreira L, et al. Regulation of Epithelial Plasticity determines Metastatic Organotropism in Pancreatic Cancer. Dev Cell. 2018; 45(6): 696-711.

9. Crawford HC, Pasca di Magliano M, Banerjee S. Signaling Networks That Control Cellular Plasticity in Pancreatic Tumorigenesis, Progression, and Metastasis. Gastroenterology. 2019; 156(7): 2073-2084.

10. Oweira H, Petrausch U, Helbling D, et al. Prognostic value of site-specific metastases in pancreatic adenocarcinoma: A Surveillance Epidemiology and End Results database analysis. World J Gastroenterol. 2017; 23: 1872-1880.

11. Allen PJ, Kuk D, Castillo CF, et al. Multi-institutional Validation Study of the American Joint Commission on Cancer (8th Edition) Changes for $\mathrm{T}$ and $\mathrm{N}$ Staging in Patients With Pancreatic Adenocarcinoma. Ann Surg. 2017; 265: 185-191.

12. Rochefort P, Lardy-Cleaud A, Sarabi M, et al. Long-Term Survivors in Metastatic Pancreatic Ductal Adenocarcinoma: A Retrospective and Matched Pair Analysis. Oncologist. 2019; 24(12): 1543-1548.

13. Tabernero J, Chiorean EG, Infante JR, et al. Prognostic factors of survival in a randomized phase III trial (MPACT) of weekly nab-paclitaxel plus gemcitabine versus gemcitabine alone in patients with metastatic pancreatic cancer. Oncologist. 2015; 20: 143-150.

14. Yamashita K, Miyamoto A, Hama N, et al. Survival Impact of Pulmonary Metastasis as Recurrence of Pancreatic Ductal Adenocarcinoma. Dig Surg. 2015; 32: 464-471.

15. Adamska A, Falasca M. Epithelial plasticity is crucial for pancreatic cancer metastatic organotropism. Ann Transl Med. 2018; 6: S53.

16. Guinney J, Wang T, Laajala TD, et al. Prediction of overall survival for patients with metastatic castration-resistant prostate cancer: development of a prognostic model through a crowdsourced challenge with open clinical trial data. Lancet Oncol. 2017; 18: 132-142.

17. Rougier P, Riess $H$, Manges $\mathrm{R}$, et al. Randomised, placebo-controlled, double-blind, parallel-group phase III study evaluating aflibercept in patients receiving first-line treatment with gemcitabine for metastatic pancreatic cancer. Eur J Cancer. 2013; 49: 2633-2642.

18. Poplin E, Wasan H, Rolfe L, et al. Randomized, multicenter, phase II study of CO-101 versus gemcitabine in patients with metastatic pancreatic ductal adenocarcinoma: including a prospective evaluation of the role of hENT1 in gemcitabine or CO-101 sensitivity. J Clin Oncol. 2013; 31: 4453-4461.

19. Lu F, Poruk KE, Weiss MJ. Surgery for oligometastasis of pancreatic cancer. Chin J Cancer Res. 2015; 27: 358-367.

20. Humphris JL, Chang DK, Johns AL, et al. The prognostic and predictive value of serum CA19.9 in pancreatic cancer. Ann Oncol. 2012; 23: 1713-1722.

21. Vestergaard EM, Hein HO, Meyer H, et al. Reference values and biological variation for tumor marker CA 19-9 in serum for different Lewis and secretor genotypes and evaluation of secretor and Lewis genotyping in a Caucasian population. Clin Chem. 1999; 45: 54-61.

22. Deeb A, Haque SU, Olowokure O. Pulmonary metastases in pancreatic cancer, is there a survival influence? J Gastrointest Oncol. 2015; 6: E48-51.

23. Katz $\mathrm{MH}$, Wang $\mathrm{H}$, Fleming JB, et al. Long-term survival after multidisciplinary management of resected pancreatic adenocarcinoma. Ann Surg Oncol. 2009; 16: 836-847.

24. Kandel P, Wallace MB, Stauffer J, et al. Survival of Patients with Oligometastatic Pancreatic Ductal Adenocarcinoma Treated with Combined Modality Treatment Including Surgical Resection: A Pilot Study. J Pancreat Cancer. 2018; 4: 88-94.

25. Xu Z, Yang $Q$, Chen $X$, et al. Clinical associations and prognostic value of site-specific metastases in non-small cell lung cancer: A population-based study. Oncol Lett. 2019; 17: 5590-5600.

26. Li J, Zhu H, Sun L. Prognostic value of site-specific metastases in lung cancer: A population based study. J Cancer. 2019; 10: 3079-3086. 